\title{
Evaluation of a Generalized Elliptic-Type Integral ${ }^{1}$
}

\author{
Leo F. Epstein ${ }^{2}$ and J. H. Hubbell
}

(October 23, 1962)

Integrals of the form

$$
\Omega_{j}(k) \equiv \int_{0}^{\pi}\left[1-k^{2} \cos \phi\right]^{-\left(j+\frac{1}{2}\right)} d \phi
$$

where $0 \leq k<1$, and $j$ is a positive integer, occur in a radiation field problem. Expressions for $\Omega_{0}(k)$ and $\Omega_{1}(k)$ have been derived in terms of complete elliptic integrals of the first and second kinds. Using these values, and the recursion formula

$$
(2 j-1)\left(1-k^{4}\right) \Omega_{j}(k)=4(j-1) \Omega_{j-1}(k)-(2 j-3) \Omega_{j-2}(k)
$$

$\Omega_{j}(k)$ can be found for all values of $j$ and $k$. A number of useful series expansions and other relations are given for $\Omega_{j}(k)$, and tables are included for $0 \leq j(1) \leq 9$ and $0 \leq k^{2}(0.01) \leq 0.99$.

\section{Introduction}

In calculating the radiation field off-axis from a uniform circular disk radiating according to an arbitrary angular distribution law, it has been convenient $[1]^{3}$ to use the method of Legendre polynomial expansion of the source-detector geometry [2]. Evaluation of the coefficients in these expansions leads to a family of integrals

$$
\Omega_{j}(k) \equiv \int_{0}^{\pi}\left[1-k^{2} \cos \phi\right]^{-\left(j+\frac{1}{2}\right)} d \phi
$$

where $0 \leq k<1$. This function has a singularity at $k=1$ which corresponds, in the problem described above, to the detector at the edge of the disk.

The integral (1) is closely related to the complete elliptic integrals of the first and second kind. Thus it can be shown readily that [3, eq 291.00]

where

$$
\Omega_{0}(k)=(\sqrt{2} \kappa / k) K(\kappa)
$$

$$
\kappa^{2}=2 k^{2} /\left(1+k^{2}\right)
$$

and $K(\kappa)$ is the complete elliptic integral of the first kind [3, eq 110.06]

$$
K(\kappa) \equiv \int_{0}^{\pi / 2}\left(1-\kappa^{2} \sin ^{2} \phi\right)^{-\frac{1}{2}} d \phi
$$

Similarly, $\Omega_{1}(k)$ can be expressed [3, eq 291.01] as

$$
\Omega_{1}(k)=(\sqrt{2} \kappa / k)\left[1 /\left(1-k^{2}\right)\right] E(\kappa)
$$

where [3, eq 110.07]

$$
E(\kappa) \equiv \int_{0}^{\pi / 2}\left(1-\kappa^{2} \sin ^{2} \phi\right)^{+\frac{1}{2}} d \phi
$$

\footnotetext{
1 Work supported jointly by the Office of Civil Defense (DOD), the Navy Bureau of Yards and Docks, and the Defense Atomic Support Agency.

2 General Electric Company, Atomic Power Equipment Department, Vallecitos Atomic Laboratory, Pleasanton, Calif.

3 Figures in brackets indicate the literature references at the end of this paper.
} 
$\Omega_{j}(k)$ for $j \geq 2$ can also be developed in terms of these functions. Thus, using the integration by parts formula given by Peirce-Foster [4, eq 317], it follows at once that

$$
(2 j-1)\left(1-k^{4}\right) \Omega_{j}(k)=4(j-1) \Omega_{j-1}(k)-(2 j-3) \Omega_{j-2}(k) .
$$

This recursion formula is exact, and by using it and values of $K(\kappa)$ and $E(\kappa), \Omega_{j}(k)$ can be obtained for all values of $j$. For example, it is found by inserting (2) and (5) in (7) that

and

$$
\Omega_{2}(k)=(\sqrt{2} \kappa / k)\left[(1 / 3)\left(1-k^{4}\right)\right]\left[4 E(\kappa) /\left(1-k^{2}\right)-K(\kappa)\right]
$$

$$
\Omega_{3}(k)=(\sqrt{2} \kappa / k)\left[(1 / 15)\left(1-k^{4}\right)^{2}\right]\left[+\left(23+9 k^{4}\right) E(\kappa) /\left(1-k^{2}\right)-8 K(\kappa)\right] .
$$

It is clear that continuing in the same way $\Omega_{j}(k)$ can be obtained for all values of $j$ in terms of $K$ and $E$. Extensive tables of these complete elliptic integrals exist [3, 5, and 6 ] in addition to excellent polynomial approximations suitable for use on a high speed computer, due to Hastings [7], and a large number of exact and approximate analytical formulations [6, 8].

For values of $k^{2}$ approaching unity, the expansions (see, e.g., [5, eqs 773.3 and 774.3])

$$
K(\kappa)=\ln \left(4 / \kappa^{\prime}\right)+(1 / 4)\left\{\ln \left(4 / \kappa^{\prime}\right)-1\right\} \kappa^{\prime 2}+\ldots
$$

and

$$
E(\kappa)=1+(1 / 2)\left\{\ln \left(4 / \kappa^{\prime}\right)-1 / 2\right\}_{\kappa^{\prime 2}}+\ldots .
$$

where $\kappa^{\prime}=\sqrt{1-\kappa^{2}}$, can be used to compute $\Omega_{0}(k)$ and $\Omega_{1}(k)$ to high precision.

This technique for finding $\Omega_{j}(k)$ has several serious disadvantages, however; e.g., (a) to find $\Omega_{j}$ from (7), particularly for large values of $j$, the precision of the $\Omega_{j}$ thus computed may be poor due to the buildup of round-off errors; (b) in order to find $\Omega_{j}$ for a given $k$, it is first required that $\Omega_{0}, \Omega_{1}, \Omega_{2}, \ldots \Omega_{j-1}$ be known. If only a few values of $\Omega_{j}(k)$ are required, this method may be painfully long and complicated. For these and similar reasons, alternate methods for calculating $\Omega_{j}(k)$ are considered below.

As will also appear in the sections which follow, the quantities $\Omega_{j}(k)$ are related to an interesting collection of transcendental functions. In addition to the elliptic integrals above, it will be shown that $\Omega_{j}(k)$ is expressible in terms of Bessel functions with a purely imaginary argument, and in terms of error functions. The analysis of these functions thus involves a fascinating excursion into a number of curious bypaths in higher mathematical analysis, in addition to the practical application to the physical problem mentioned in the opening sentences above.

\section{Series and Other Expansions for $\Omega_{j}(k)$}

\subsection{Simple Power Series $j \geq 0$}

For small values of $k, \Omega_{j}(k)$ can be derived by a binomial theorem expansion of $\left[1-k^{2} \cos \phi\right]^{-\left(j+\frac{1}{2}\right)}$ in powers of $\left(k^{2} \cos \phi\right)$, i.e.,

$$
\Omega_{j}(k)=\sum_{i=0}^{\infty}\left(\begin{array}{c}
-j-\frac{1}{2} \\
i
\end{array}\right)\left(-k^{2}\right)^{i} \int_{0}^{\pi} \cos ^{i} \phi d \phi
$$

where

$$
\left(\begin{array}{c}
n \\
i
\end{array}\right) \equiv n ! / i !(n-i) !
$$

is the familiar notation for the binomial coefficient ${ }^{4}[9$, par. 1.51], [10]. The integral in (1) can be shown to be [4, eq 498]

${ }^{4}$ As indicated in [9] and [10], binomial coefficients of the form $\left(\begin{array}{c}n / 2 \\ m\end{array}\right)$ are easily generated using the recursions:

$$
\left(\begin{array}{c}
n / 2 \\
0
\end{array}\right)=1 ;\left(\begin{array}{c}
n / 2 \\
m
\end{array}\right)=\left(\begin{array}{c}
n / 2 \\
m-1
\end{array}\right)(n-2 m+2) /(2 m)=\left(\begin{array}{c}
n / 2-1 \\
m-1
\end{array}\right)+\left(\begin{array}{c}
n / 2-1 \\
m
\end{array}\right) .
$$




$$
\int_{0}^{\pi} \cos ^{i} \phi d \phi=\left[1+(-1)^{i}\right](\sqrt{\pi} / 2) \frac{\Gamma\left(\frac{i+1}{2}\right)}{\Gamma\left(\frac{i+2}{2}\right)}
$$

and so vanishes when $i$ is odd. Using this relation and the well known properties of the binomial coefficients, it follows that

$$
\Omega_{j}(k)=\sum_{m=0}^{\infty} \alpha_{m}(j) k^{4 m}
$$

where

$$
\alpha_{m}(j)=\frac{\pi}{(64)^{m}} \frac{j !}{(2 j) !} \frac{(4 m+2 j) !}{(2 m+j) !}\left(\frac{1}{m !}\right)^{2}
$$

Equation (4) may easily be shown to be convergent for all values of $j$ and for $k \leq 1$. For $k$ close to unity, however, it tends to converge rather slowly; and for this reason, this power series formulation is most useful for $k<<1$.

\subsection{Difference Sum, $j \geq 0$}

The behavior of (4) may be improved by using a technique previously developed for improving the convergence of series [8]. By this method, the series (4) is written in the identically equivalent form

$$
\Omega_{j}(k)=\sum_{m=0}^{\infty} \alpha_{m}(j) k^{4 m}=\sum_{m=0}^{\infty} \alpha_{m}^{*}(j) k^{4 m}+\sum_{m=0}^{\infty}\left[\alpha_{m}(j)-\alpha_{m}^{*}(j)\right] k^{4 m}
$$

where $\alpha_{m}^{*}(j)$ is defined, first, by the condition

$$
\lim _{m \rightarrow \infty}\left[\alpha_{m}^{*}(j) / \alpha_{m}(j)\right]=1
$$

that is, $\alpha_{m}^{*}$ is asymptotically equivalent to $\alpha_{m}$. From the infinite variety of sets of $\alpha_{m}^{*}$ values satisfying this condition, a set is chosen such that the first sum on the right of eq (6) can be evaluated in closed form. If this technique is properly applied, the residual sum of the differences $\left[\alpha_{m}(j)-\alpha_{m}^{*}(j)\right] k^{4 m}$ converges much more rapidly than the original sum in $\alpha_{m}(j) k^{4 m}$.

For the present problem, it is shown in appendix A that by substituting Stirling's formula $\left[11\right.$, p. 5] for the factorials in eq (5) such a set of $\alpha_{m}^{*}(j)$ 's can be generated, of the form

$$
\alpha_{m}^{*}(j)=A_{j}\left\{\sum_{i \geq 0}^{j-1} B_{i j}\left(\begin{array}{c}
m+j-i-1 \\
m
\end{array}\right)+\frac{1}{m+1}\right\}
$$

where

$$
A_{j}=(1 / \sqrt{2}) 2^{-3 j}\left(\begin{array}{c}
2 j \\
j
\end{array}\right)
$$

and

$$
B_{i j}=(-1)^{j+i} 2^{2 j-2 i}\left(\begin{array}{l}
j \\
i
\end{array}\right) /\left[\left(\begin{array}{c}
2 j-2 i \\
j-i
\end{array}\right)\left(\begin{array}{c}
j-\frac{1}{2} \\
2 j-2 i
\end{array}\right)(j-i)\right]
$$

The notation $\sum_{i \geq 0}^{j-1}$ means that the finite sum over $i$ vanishes when $j=0$.

Using (8), sums over $\alpha_{m}^{*}(j) k^{4 m}$ can then be identified with the closed-form expressions

$$
\sum_{m=0}^{\infty} \alpha_{m}^{*}(j) k^{4 m}=A_{j}\left\{\sum_{i \geq 0}^{j-1} B_{i j}\left[1 /\left(1-k^{4}\right)\right]^{j-i}+\left(-1 / k^{4}\right) \ln \left(1-k^{4}\right)\right\} .
$$

From (8) we have, for $j=0,1$ and 2

$$
\alpha_{m}^{*}(0)=(1 / \sqrt{2}) \frac{1}{m+1},
$$




$$
\alpha_{m}^{*}(1)=(1 / \sqrt{2})\left\{4+(1 / 4) \frac{1}{m+1}\right\}
$$

and

$$
\alpha_{m}^{*}(2)=(1 / \sqrt{2})\left\{(16 / 3)(m+1)-1+(3 / 32) \frac{1}{m+1}\right\} .
$$

The corresponding sums from (11) are

$$
\begin{gathered}
\sum_{m=0}^{\infty} \alpha_{m}^{*}(0) k^{4 m}=(1 / \sqrt{2})\left(-1 / k^{4}\right) \ln \left(1-k^{4}\right), \\
\sum_{m=0}^{\infty} \alpha_{m}^{*}(1) k^{4 m}=(1 / \sqrt{2})\left\{4 /\left(1-k^{4}\right)+(1 / 4)\left(-1 / k^{4}\right) \ln \left(1-k^{4}\right)\right\},
\end{gathered}
$$

and

$$
\sum_{m=0}^{\infty} \alpha_{m}^{*}(2) k^{4 m}=(1 / \sqrt{2})\left\{(16 / 3) /\left(1-k^{4}\right)^{2}-1 /\left(1-k^{4}\right)+(3 / 32)\left(-1 / k^{4}\right) \ln \left(1-k^{4}\right)\right\} .
$$

Using (5) and the particular form of $\alpha_{m}^{*}(j)$ given in (8), the residual sum in (6), viz,

$$
\sum_{R^{\prime}}=\sum_{m=0}^{\infty}\left[\alpha_{m}(j)-\alpha_{m}^{*}(j)\right] k^{4 m}
$$

converges as fast as $\sum_{m=0}^{\infty}\left[1 /(m+1)^{2}\right] k^{4 m}$. This may be contrasted with the simple power series (4) which only converges as fast as $\sum_{m=0}^{\infty} m^{j-1} k^{4 m}$.

An additional bonus is noted for the higher values of $j$. While $\Sigma_{R}$ for any given $j$ varies by less than a factor of two over the entire range $0 \leq k^{2} \leq 1$, the total value of the function $\Omega_{j}(k)$ varies from $\pi$ up to infinity over this same range. Thus, in the region of weakest convergence of $\Sigma_{R}$, i.e., $0.90 \lesssim k^{2} \leq 1$, the contribution of $\Sigma_{R}$ to $\Omega_{j}(k)$ may be neglected in many cases.

Also, in the right hand side of (11), the successive terms in the finite sum decrease very rapidly with increasing $i$, and the log-term, $\left(-1 / k^{4}\right) \ln \left(1-k^{4}\right)$, is, for $j \geq 1,0 \leq k^{2} \leq 1$, of the same order of magnitude or less than the final term $(i=j-1)$ in the finite sum. Thus, for example, for $j \geq 6, k^{2} \geq 0.90$, values of $\Omega_{j}(k)$ accurate to one part in $10^{8}$ may be computed by omitting from (11) the finite series terms for $i \geq 5$, the log-term $\left(-1 / k^{4}\right) \ln \left(1-k^{4}\right)$, and the entire sum $\Sigma_{R}$ on the right side of $(6)$.

This ease of attaining $\Omega_{j}(k)$ values of high precision for large $j$ proved useful in evaluating disk-source Legendre coefficients [1] for large $l$, which involve smaller and smaller differences between larger and larger numbers.

\section{3. $\Omega_{j}(k)$ in Terms of Bessel Functions, $j \geq 0$}

The differencing technique illustrated for the sum in section 2.2 above may often profitably be applied to integrals as well, i.e., by writing

$$
\int_{a}^{b} f(\phi) d \phi=\int_{a}^{b} f^{*}(\phi) d \phi+\int_{a}^{b}\left[f(\phi)-f^{*}(\phi)\right] d \phi .
$$

In this expression $f^{*}(\phi)$ is a function, integrable in closed form over the region $a \leq \phi \leq b$, which is a suitable approximation to $f(\phi)$. Applied to $\Omega_{j}(k)$, it will be noted from $(1-1)$ that

$$
f(\phi)=\left(1-k^{2} \cos \phi\right)^{-n}
$$

where

$$
n=j+\frac{1}{2}
$$


and

For this case, it is convenient to take

$$
a=0, b=\pi \text {. }
$$

$$
f^{*}(\phi)=e^{+n\left(k^{2} \cos \phi\right)}+(n / 2)\left(k^{2} \cos \phi\right)^{2} e^{+(n+2 / 3)\left(k^{2} \cos \phi\right)},
$$

an expression derived by retaining terms through $\left(k^{2} \cos \phi\right)^{3}$ in the expansion of $f(\phi)$ as given by (19). Then

$$
\int_{0}^{\pi} f^{*}(\phi) d \phi=\int_{0}^{\pi} \exp \left[+n^{2}\left(k^{2} \cos \phi\right)\right] d \phi+(n / 2) k^{2} \int_{0}^{\pi}\left(\cos ^{2} \phi\right) \exp \left[\left(n+\frac{2}{3}\right)\left(k^{2} \cos \phi\right)\right] d \phi .
$$

Now from the expression [12, p. 79, No. 9]

$$
I_{\nu}(z)=\frac{(z / 2)^{\nu}}{\Gamma\left(\nu+\frac{1}{2}\right) \Gamma\left(\frac{1}{2}\right)} \int_{0}^{\pi}\left(\sin ^{2} \phi\right)^{\nu}[ \pm z \cos \phi] d \phi,
$$

where $I_{\nu}(z)$ is the Bessel function of order $\nu$ with a purely imaginary argument, i.e.,

From $(24)$

$$
I_{\nu}(z) \equiv i^{-\nu} J_{\nu}(i z) .
$$

and

$$
I_{0}(z)=(1 / \pi) \int_{0}^{\pi} \exp [ \pm z \cos \phi] d \phi
$$

$$
I_{1}(z)=(z / \pi) \int_{0}^{\pi}\left(\sin ^{2} \phi\right) \exp [ \pm z \cos \phi] d \phi
$$

Using (26) to evaluate the integrals in (23), it is found that

$$
\begin{gathered}
(1 / \pi) \Omega_{j}(k)=+I_{0}\left[k^{2}\left(j+\frac{1}{2}\right)\right]-\left(\frac{3}{2}\right) k^{2}\left(\frac{2 j+1}{6 j+7}\right) I_{1}\left[k^{2}\left(j+\frac{7}{6}\right)\right]+\left(\frac{1}{4}\right) k^{4}(2 j+1) I_{0}\left[k^{2}\left(j+\frac{7}{6}\right)\right] \\
+(1 / \pi) \int_{0}^{\pi}\left[\left(1-k^{2} \cos \phi\right)^{-\left(j+\frac{1}{2}\right)}-\exp \left\{+\left(j+\frac{1}{2}\right)\left(k^{2} \cos \phi\right)\right\}\right. \\
\left.\quad-\left(\frac{1}{2}\right)\left(j+\frac{1}{2}\right)\left(k^{2} \cos \phi\right)^{2} \exp \left\{\left(j+\frac{7}{6}\right)\left(k^{2} \cos \phi\right)\right\}+\ldots\right] d \phi .
\end{gathered}
$$

By expanding

$$
(1-x)^{-n}-e^{+n x}-\frac{1}{2} n x^{2} e^{+\left(n+\frac{2}{3}\right) x}
$$

in a power series in $x=k^{2} \cos \phi$ and integrating term by term, the integral in (27) may be shown to be equal to

$$
\begin{aligned}
\sum_{m=2}^{\infty}(1 / m !)^{2}\left[+\frac{(2 j+4 m) !}{(j+2 m) !} \cdot \frac{j !}{(2 j) !}-4^{m}(2 j+1)^{2 m}-18(2 / 3)^{2 m}\right. & \left.\cdot m(2 m-1)(2 j+1)(6 j+7)^{2 m-2}\right]\left(k^{4} / 64\right)^{m} \\
& =(3 / 64)\left(j+\frac{1}{2}\right)\left(j+\frac{29}{18}\right) k^{8}+\ldots
\end{aligned}
$$

Further manipulation and simplification in the case of (28) is possible, but these procedures have not proven particularly rewarding. The greatest importance of eq (27), which it will be observed is exact, arises from the relation shown by this equation between $\Omega_{j}(k)$ and the Bessel functions. For numerical calculation, forms other than (27) and (28) may be more convenient, except possibly for some special cases.

It will be noted that $f^{*}(\phi)$ as given by $(22)$ is a good approximation to $f(\phi)$ only for small values of $k^{2}$, since it is derived from the first three terms of the power series expansion of (19). Consequently, (27) is essentially useful only for $|k|<<1$. To realize the full potentialities of the formulation (18), it would be necessary to find a form for $f^{*}(\phi)$ valid over the whole range $0<k<1$ and integrable in closed form. Such an approximation is explored in section 2.5 below. 
By writing

so that

$$
\Omega_{j}(k) \equiv \int_{0}^{\pi} e^{-F(\phi)} d \phi
$$

$$
F(\phi)=\left(j+\frac{1}{2}\right) \ln \left(1-k^{2} \cos \phi\right)
$$

it is possible to obtain another interesting form for the function. Expanding $F(\phi)$ in a Maclaurin series [9, par. 6.104], it can readily be shown that all the odd derivatives of $F(\phi)$ vanish at the origin and that

where

$$
\Omega_{j}(k)=e^{-F^{(0)}} \int_{0}^{\pi} e^{-(\lambda / \pi)^{2} \phi^{2}}\left[1-(1 / 4 !) F^{(4)}(0) \phi^{4}-(1 / 6 !) F^{(6)}(0) \phi^{6}+\ldots .\right] d \phi
$$

$$
(\lambda / \pi)^{2}=(1 / 2) F^{(2)}(0) .
$$

Inserting the value of $F(0)$ and making the transformation $t=(\lambda / \pi) \phi$, it follows at once that

$$
\Omega_{j}(k)=(\pi / \lambda)\left(1-k^{2}\right)^{-\left(j+\frac{1}{2}\right)}\left[L_{0}(\lambda)-(1 / 4 !) F^{(4)}(0)(\pi / \lambda)^{4} L_{2}(\lambda)-(1 / 6 !) F^{(6)}(0)(\pi / \lambda)^{6} L_{3}(\lambda)+\ldots\right]
$$

where

$$
L_{p}(x) \equiv \int_{0}^{x} t^{2 p} e^{-t^{2}} d t
$$

The integrals $L_{p}(x)$ can be expressed in terms of the incomplete gamma [13] or factorial [11] functions, $L_{p}(x)=\frac{1}{2} \cdot\left(p-\frac{1}{2}, x^{2}\right)$ ! but an alternate procedure is perhaps more informative. Integrating (32) by parts, from the resulting recursion formula

$$
L_{p+1}(x)=(1 / 2)\left[(2 p+1) L_{p}(x)-x^{2 p+1} e^{-x^{2}}\right]
$$

the values of $L_{p}(x)$ can all be found in terms of $L_{0}(x)$. But this is simply

$$
L_{0}(x)=(\sqrt{\pi} / 2) \operatorname{erf} x
$$

where the error function [5, par. 5.90] is

$$
\operatorname{erf} x \equiv(2 / \sqrt{\pi}) \int_{0}^{x} e^{-t^{2}} d t .
$$

By this method then, it is found that

$$
\begin{aligned}
& L_{2}(x)=(3 / 4)\left[L_{0}(x)-x e^{-x^{2}}\left(1+\frac{2}{3} x^{2}\right)\right] \\
& L_{3}(x)=(15 / 8)\left[L_{0}(x)-x e^{-x^{2}}\left(1+\frac{2}{3} x^{2}+\frac{4}{15} x^{4}\right)\right] .
\end{aligned}
$$

The higher derivatives of $F(\phi)$ at the origin, $F^{(p)}(0)$, are readily determined by expanding $F(\phi)$ in a power series and recognizing that $F^{(p)}(0)$ is $p$ ! times the coefficient of $\phi^{p}$. In this way, it is found that

From $(36 \mathrm{~A})$ and $(30)$,

$$
\begin{aligned}
& F^{(2)}(0)=+\left(j+\frac{1}{2}\right) k^{2}\left(1-k^{2}\right)^{-1} \\
& F^{(4)}(0)=-\left(j+\frac{1}{2}\right) k^{2}\left(1-k^{2}\right)^{-2}\left[2 k^{2}+1\right] \\
& F^{(6)}(0)=+\left(j+\frac{1}{2}\right) k^{2}\left(1-k^{2}\right)^{-3}\left[16 k^{4}+13 k^{2}+1\right] .
\end{aligned}
$$

$$
\lambda=(\pi / 2) \sqrt{(2 j+1) k^{2} /\left(1-k^{2}\right)}
$$


and combining this and the previous results with (31), it follows that

$$
\begin{aligned}
\Omega_{j}(k)= & \sqrt{\pi /(2 j+1) k^{2}}\left(1-k^{2}\right)^{-j}\left[+\operatorname{erf} \lambda+(1 / 2)(2 j+1)^{-1}\left(1+\frac{1}{2 k^{2}}\right)\left\{\operatorname{erf} \lambda-(2 / \sqrt{\pi})\left(\lambda e^{-\lambda^{2}}\right)\left(1+\frac{2}{3} \lambda^{2}\right)\right\}\right. \\
& \left.-(1 / 12)(2 j+1)^{-2}\left(16+\frac{13}{k^{2}}+\frac{1}{k^{4}}\right)\left\{\operatorname{erf} \lambda-(2 / \sqrt{\pi})\left(\lambda e^{-\lambda^{2}}\right)\left(1+\frac{2}{3} \lambda^{2}+\frac{4}{15} \lambda^{4}\right)\right\}+\ldots\right] .
\end{aligned}
$$

In using these relations for small $k^{2}$ or $\lambda$ values, the problem of small differences between large quantities is resolved by the series

$$
\begin{aligned}
\left\{\operatorname{erf} \lambda-(2 / \sqrt{\pi})\left(\lambda e^{-\lambda^{2}}\right)\left(1+\frac{2}{3} \lambda^{2}\right)\right\}=(2 / \sqrt{\pi})\left(4 \lambda^{5} / 15\right) & \left\{1-(5 / 7) \lambda^{2}+(5 / 18) \lambda^{4}-(5 / 66) \lambda^{6}\right. \\
& \left.+(5 / 312) \lambda^{8}-(1 / 360) \lambda^{10}+(1 / 2448) \lambda^{12}+\ldots\right\}
\end{aligned}
$$

and

$$
\begin{aligned}
\{\operatorname{erf} \lambda & \left.-(2 / \sqrt{\pi})\left(\lambda e^{-\lambda^{2}}\right)\left(1+\frac{2}{3} \lambda^{2}+\frac{4}{15} \lambda^{4}\right)\right\} \\
= & (2 / \sqrt{\pi})\left(8 \lambda^{7} / 105\right)\left\{1+(77 / 16) \lambda^{2}+(7 / 22) \lambda^{4}-(7 / 78) \lambda^{6}+(7 / 360) \lambda^{8}-(7 / 2040) \lambda^{10}+\ldots\right\} .
\end{aligned}
$$

For large $\lambda$ values, i.e., large $j$ 's, erf $\lambda$ and the bracket terms involving this quantity are all close to unity, and

$$
\begin{aligned}
\lim _{j \rightarrow \infty} \Omega_{j}(k)=\sqrt{\pi /(2 j+1) k^{2}}\left(1-k^{2}\right)^{-j}\left[1+(1 / 2)(2 j+1)^{-1}\left\{1+\frac{1}{2 k^{2}}\right\}\right. & \\
& \left.-(4 / 3)(2 j+1)^{-2}\left\{1+\frac{13}{16 k^{2}}+\frac{1}{16 k^{4}}\right\}+\ldots\right] .
\end{aligned}
$$

\subsection{Numerical Integration Techniques for $\Omega_{j}(k)$}

To apply eq (18) in a more general way than was used in section 2.3 above, let

$$
f^{*}(\phi)=\left(1-k^{2}\right)^{-n}
$$

where $n=j+1 / 2$, as before (eq 20). Then from (18)

$$
\Omega_{j}(k)=\pi\left(1-k^{2}\right)^{-n}+\int_{0}^{\pi}\left[\left(1-k^{2} \cos \phi\right)^{-n}-\left(1-k^{2}\right)^{-n}\right] d \phi .
$$

It will be observed that the integrand here contains no singularities, even at the origin for $k=1$. With the change of variable $\phi=\pi x$, this becomes

$$
\Omega_{j}(k)=\pi\left(1-k^{2}\right)^{-n}+\pi \int_{0}^{1}\left[\left\{1-k^{2} \cos (\pi x)^{-n}\right\}-\left(1-k^{2}\right)^{-n}\right] d x .
$$

This integral is now in standard form for the use of numerical integration techniques. By fitting a polynomial of the $N^{\prime}$ th degree to $\Xi(x)$, it can be shown that

$$
\int_{0}^{1} \Xi(x) d x \cong \sum_{i=0}^{N} w_{N}(i) \Xi(i / N) / \sum_{i=0}^{N} w_{N}(i)
$$

where the $w_{N}(i)$ 's are constants (given for example in the introduction to the Tables of Lagrangian Interpolation Coefficients [14] where the integrals are expressed in slightly different form). It should be noted that (43) can never be expected to give exact answers; but by increasing the value of $N$, closer and closer approximations to $\Omega_{j}(k)$ can be obtained over the whole range of $k$, although this procedure does not necessarily result in improved accuracy at any given value of $k$. In the present case, where for certain values of $N, \cos (i \pi / N)$ can be expressed in simple closed form, this technique leads to an interesting series of approximations 
for $\Omega_{j}(k)$ as given below. Note that all these results yield exactly the right answers at $k=0, \Omega_{j}(0)=\pi$

$$
n=j+\frac{1}{2}
$$

$$
\begin{gathered}
N=2 \\
\Omega_{j}(k)=(\pi / 6)\left[+4+\left(1-k^{2}\right)^{-n}+\left(1+k^{2}\right)^{-n}\right]
\end{gathered}
$$

$$
\begin{gathered}
N=3 \\
\Omega j(k)=(\pi / 8)\left[+\left(1-k^{2}\right)^{-n}+\left(1+k^{2}\right)^{-n}+3\left(1-\frac{1}{2} k^{2}\right)^{-n}+3\left(1+\frac{1}{2} k^{2}\right)^{-n}\right]
\end{gathered}
$$

$$
\begin{gathered}
N=4 \\
\Omega_{j}(k)=(\pi / 90)\left[+12+7\left(1-k^{2}\right)^{-n}+7\left(1+k^{2}\right)^{-n}+32\left(1-\frac{1}{\sqrt{2}} k^{2}\right)^{-n}+32\left(1+\frac{1}{\sqrt{2}} k^{2}\right)^{-n}\right]
\end{gathered}
$$

$$
N=5
$$

$\Omega_{j}(k)=(\pi / 288)\left[+19\left(1-k^{2}\right)^{-n}+19\left(1+k^{2}\right)^{-n}+75\left(1-\frac{\sqrt{5}+1}{4} k^{2}\right)^{-n}\right.$

$$
\left.+75\left(1+\frac{\sqrt{5}+1}{4} k^{2}\right)^{-n}+50\left(1-\frac{\sqrt{5}-1}{4} k^{2}\right)^{-n}+50\left(1+\frac{\sqrt{5}-1}{4} k^{2}\right)^{-n}\right]
$$

$$
N=6
$$

$\Omega_{j}(k)=(\pi / 840)\left[+272+41\left(1-k^{2}\right)^{-n}+41\left(1+k^{2}\right)^{-n}+216\left(1-\frac{1}{2} \sqrt{3} k^{2}\right)^{-n}\right.$

$$
\left.+216\left(1+\frac{1}{2} \sqrt{3} k^{2}\right)^{-n}+27\left(1-\frac{1}{2} k^{2}\right)^{-n}+27\left(1+\frac{1}{2} k^{2}\right)^{-n}\right]
$$

$$
\begin{aligned}
& N=10 \\
& \Omega_{j}(k)=(\pi / 598,752)\left[+427,368+16,067\left(1-k^{2}\right)^{-n}+16,067\left(1+k^{2}\right)^{-n}\right. \\
& +106,300\left(1-\sqrt{\frac{5+\sqrt{5}}{8}} k^{2}\right)^{-n}+106,300\left(1+\sqrt{\frac{5+\sqrt{5}}{8}} k^{2}\right)^{-n}-48,525\left(1-\frac{\sqrt{5}+1}{4} k^{2}\right)^{-n} \\
& -48,525\left(1+\frac{\sqrt{5}+1}{4} k^{2}\right)^{-n}+272,400\left(1-\sqrt{\frac{5-\sqrt{5}}{8}} k^{2}\right)^{-n}+272,400\left(1+\sqrt{\frac{5-\sqrt{5}}{8}} k^{2}\right)^{-n} \\
& \left.-260,550\left(1-\frac{\sqrt{5}-1}{4} k^{2}\right)^{-n}-260,550\left(1+\frac{\sqrt{5}-1}{4} k^{2}\right)^{-n}\right] \text {. }
\end{aligned}
$$

The interesting and beautiful symmetries in these approximations have been emphasized in the formulas above. It is evident that by using a more complicated expression for $f^{*}(\phi)$ than (42), better agreement can be achieved. Also, by going to larger values of $N$, or by dividing the range of $\phi$ into smaller intervals, formulas of greater accuracy can be developed. For large values of $j$ and $k^{2}$, or their product

$$
\lim _{j k^{2} \rightarrow \infty} \Omega_{j}(k)=\pi\left(1-k^{2}\right)^{-\left(j+\frac{1}{2}\right)} w_{N}(0) / \sum_{i=0}^{N} w_{N}(i)
$$


or inserting the expression from $w_{N}(i)$ given by Whittaker and Robinson [15]

$$
\lim _{j k^{2} \rightarrow \infty} \Omega_{j}(k)=\pi\left(1-k^{2}\right)^{-\left(j+\frac{1}{2}\right)} \cdot \frac{(-1)^{N}}{N} \int_{0}^{N}\left(\begin{array}{c}
t-1 \\
N
\end{array}\right) d t
$$

where, it will turn out, since the $w_{N}(i)$ 's are all integers, the last term here is a rational fraction.

\section{Numerical}

It is immediately apparent from the definition of the function that

$$
\Omega_{j}(0)=\pi \text { and } \Omega_{j}(1)=\infty .
$$

The intermediate behavior of $\Omega_{j}(k)$ versus $k^{2}$ is displayed for $0 \leq j(1) \leq 9$ as a family of curves in figure 1. These curves are based on values of $\Omega_{j}(k)$ listed for $0 \leq j(1) \leq 9$ and $0 \leq k^{2}(0.01) \leq$ 0.99 in table 1.

Table 1 was computed in "double precision" on the National Bureau of Standards IBM 7090 electronic computing machine, using the difference-sum (2.2-6) with the particular $\alpha_{m}^{*}(j)$ given in $(2.2-8)$. The results are given to eight digits, which were rounded off from a table correct to at least nine digits throughout, so all eight digits can be considered significant. The results in the region of poorest convergence (high $k$, low $j$ ) of the difference sum were verified by a ten-digit hand calculation using the recursion expression $(1-7)$ and the high- $k$ elliptic integral expansions (1-10) and (1-11).

The lengthy developments in section 2 can be considered to yield various approximations to the value of $\Omega_{j}(k)$. In tables 2 to 6 numerical examples are presented which will give some idea of the usefulness of the techniques. For each example, the values $j=0$ and 8 , and $k^{2}=$ 0.01 and 0.99 are used for purposes of illustration.

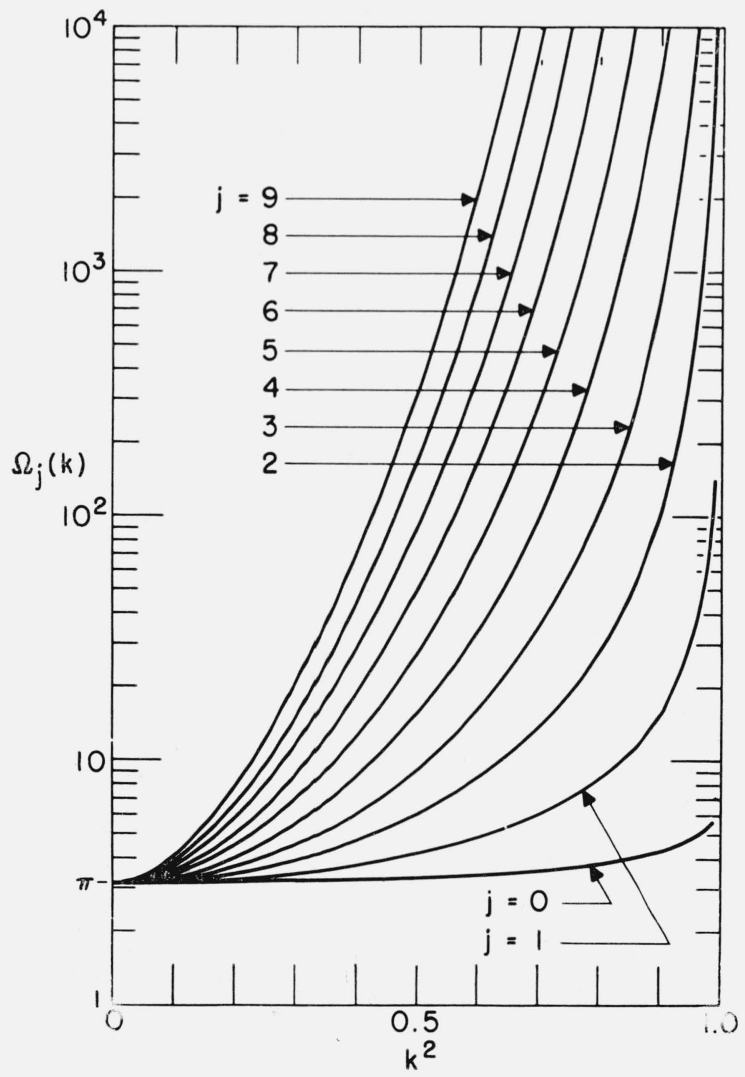

FiguRE 1. Family of $\Omega_{j}(\mathrm{k})$ curves based on numerical results in table 1 . 
TABLE 1.* Values of $\Omega_{\mathrm{j}}(\mathrm{k})$ for $0 \leq \mathrm{j}(1) \leq 9$ and $0 \leq \mathrm{k}^{2}(0.01)<1.00$

These were computed using the difference sum $(2.2-6)$ with the $\alpha_{m}^{*}(j)$ in $(2.2-8)$. A tolerance of $<10^{-11}$ was set on the fractional contribution of the first neglected series term $\left[\alpha_{m}(j)-\alpha_{m}{ }^{*}(j)\right] k^{4 m}$ which resulted in nine or more significant figures throughout, here rounded off to eight significant figures.

\begin{tabular}{|c|c|c|c|c|c|}
\hline$k^{2}$ & $\Omega_{0}$ & $\Omega_{1}$ & $\Omega_{2}$ & $\Omega_{3}$ & $\Omega_{4}$ \\
\hline 0.00 & $\pi$ & $\pi$ & $\pi$ & $\pi$ & $\pi$ \\
\hline .01 & 3. $1416516(0)$ & $3.1418872(0)$ & $3.1422800(0)$ & $3.1428299(0)$ & $3.1435371 \quad(0)$ \\
\hline .02 & 3. $1418283(0)$ & 3. $1427712(0)$ & 3. $1443432(0)$ & 3. $1465451 \quad(0)$ & 3. $1493776(0)$ \\
\hline .03 & 3. $1421231(0)$ & 3. $1442457(0)$ & 3. $1477863(0)$ & 3. 1527481 (0) & $3.1591355(0)$ \\
\hline .04 & 3. $1425360(0)$ & $3.1463125(0)$ & $3.1526155(0)$ & $3.1614556(0)$ & $3.1728466(0)$ \\
\hline .05 & 3. $1430673(0)$ & 3. $1489739(0)$ & 3. $1588399(0)$ & 3. $1726912(0)$ & 3. $1905614(0)$ \\
\hline .06 & 3. $1437174(0)$ & $3.1522332(0)$ & $3.1664711(0)$ & $3.1864852(0)$ & 3. $2123454(0)$ \\
\hline .07 & 3. $1444868(0)$ & 3. $1560943(0)$ & 3. $1755235(0)$ & 3. $2028752(0)$ & 3. $2382796(0)$ \\
\hline .08 & 3. $1453758(0)$ & 3. $1605617(0)$ & 3. $1860142(0)$ & 3. 2219059 (0) & 3. $2684609(0)$ \\
\hline .09 & 3. $1463852(0)$ & 3. $1656409(0)$ & 3. $1979629(0)$ & 3. $2436296(0)$ & $3.3030029(0)$ \\
\hline .10 & 3. $1475156(0)$ & 3. $1713379(0)$ & 3. $2113926(0)$ & 3. $2681065(0)$ & 3. $3420368(0)$ \\
\hline .11 & $3.1487677(0)$ & 3. $1776597 \quad(0)$ & 3. $2263290(0)$ & 3. $2954049(0)$ & $3.3857120(0)$ \\
\hline .12 & 3. $1501424(0)$ & 3. $1846141(0)$ & 3. 2428009 (0) & 3. 3256017 (0) & 3. $4341976(0)$ \\
\hline .13 & $3.1516407(0)$ & $3.1922094(0)$ & $3.2608406(0)$ & 3.3587827 (0) & 3. $4876832(0)$ \\
\hline .14 & 3.1532635 (0) & $3.2004553(0)$ & $3.2804834(0)$ & 3.3950431 (0) & 3. $5463805(0)$ \\
\hline .15 & 3. $1550119(0)$ & 3. $2093619(0)$ & 3. $3017684(0)$ & 3. $4344882(0)$ & 3. $6105249(0)$ \\
\hline .16 & 3. $1568872(0)$ & $3.2189405 \quad(0)$ & 3. $3247382(0)$ & $3.4772340 \quad(0)$ & $3.6803773(0)$ \\
\hline .17 & 3. $1588907(0)$ & 3. $2292032(0)$ & 3. $3494395(0)$ & 3.5234078 & 3. $7562257 \quad(0)$ \\
\hline .18 & 3. $1610237(0)$ & 3. $2401632(0)$ & 3. $3759229(0)$ & $3.5731488(0)$ & $3.8383882(0)$ \\
\hline .19 & 3. $1632878(0)$ & 3. $2518347(0)$ & 3. $4042436(0)$ & 3. $6266094(0)$ & 3. $9272147(0)$ \\
\hline .20 & 3. $1656846(0)$ & 3. $2642330(0)$ & $3.4344610(0)$ & 3. $6839559(0)$ & 4. $0230902(0)$ \\
\hline .21 & 3. $1682158(0)$ & $3.2773746(0)$ & $3.4666396(0)$ & 3. $7453695 \quad(0)$ & 4. $1264381(0)$ \\
\hline .22 & $3.1708833(0)$ & 3.2912770 (0) & 3. $5008493(0)$ & 3.8110474 (0) & 4. $23770233(0)$ \\
\hline .23 & 3. $1736890(0)$ & 3. $3059591(0)$ & 3. $5371653(0)$ & 3. $8812046(0)$ & 4. $3574565(0)$ \\
\hline .24 & 3. $1766349(0)$ & 3. $3214413(0)$ & $3.5756686(0)$ & $3.9560749(0)$ & 4. $4861987(0)$ \\
\hline .25 & 3. $1797233(0)$ & $3.3377450(0)$ & 3. $6164468(0)$ & 4. $0359125(0)$ & 4. $6245662(0)$ \\
\hline .26 & 3. $1829566(0)$ & $3.3548935 \quad(0)$ & 3. $6595943(0)$ & 4.1209941 (0) & 4. $7732360(0)$ \\
\hline .27 & 3. $1863371(0)$ & 3. $3729113(0)$ & 3.7052127 (0) & 4. 2116207 (0) & 4. $9329530(0)$ \\
\hline .28 & 3. $1898676(0)$ & 3. $3918249(0)$ & 3. $7534115(0)$ & 4. 3081200 (0) & 5. $1045360(0)$ \\
\hline .29 & 3. $1935508(0)$ & $3.4116624(0)$ & $3.8043086(0)$ & 4. 4108487 (0) & 5. 2888871 (0) \\
\hline .30 & 3. $1973897(0)$ & 3. $4324538(0)$ & 3. $8580313(0)$ & 4. 5201954 (0) & 5. $4869998(0)$ \\
\hline .31 & 3. 2013874 (0) & $3.4542312(0)$ & $3.9147167 \quad(0)$ & 4. $6365838 \quad(0)$ & 5. $6999702(0)$ \\
\hline .32 & $3.2055471 \quad(0)$ & 3. $4770287(0)$ & 3. $9745127(0)$ & 4. $7604758(0)$ & 5. $9290085(0)$ \\
\hline .33 & 3. 2098724 (0) & 3. $5008829(0)$ & 4. 0375787 (0) & 4.8923759 (0) & 6. $1754521(0)$ \\
\hline .34 & 3. $2143669(0)$ & $3.5258327(0)$ & 4. 1040871 & $5.0328355(0)$ & 6. $4407815(0)$ \\
\hline .35 & 3. $2190345(0)$ & $3.5519197(0)$ & 4. $1742238(0)$ & 5. $1824573(0)$ & 6. $7266372(0)$ \\
\hline .36 & 3. $2238793(0)$ & $3.5791883(0)$ & 4. $2481901 \quad(0)$ & $5.3419016 \quad(0)$ & 7. $0348393(0)$ \\
\hline .37 & 3. $2289056(0)$ & 3. $6076862(0)$ & 4. $3262036(0)$ & 5.5118921 & 7.3674111 (0) \\
\hline .38 & 3. $2341182(0)$ & 3. $6374640(0)$ & 4. $4085000(0)$ & 5. $6932230(0)$ & $7.7266040(0)$ \\
\hline .39 & 3. $2395217(0)$ & 3. 6685762 (0) & 4. $4953348(0)$ & $5.8867672(0)$ & 8. $1149281(0)$ \\
\hline .40 & 3. $2451213(0)$ & 3. $7010809(0)$ & 4. $5869850(0)$ & 6.0934851 (0) & 8.5351864 (0) \\
\hline .41 & 3. $2509225(0)$ & 3. $7350405 \quad(0)$ & 4. $6837518(0)$ & $6.3144352(0)$ & 8. $9905145 \quad(0)$ \\
\hline .42 & 3. 2569310 & 3. $7705219(0)$ & 4. 7859626 & 6.5507857 (0) & 9. $4844263(0)$ \\
\hline .43 & 3. $2631530(0)$ & $3.8075968(0)$ & 4. 8939738 (0) & 6.8038277 (0) & 1. $0020868(1)$ \\
\hline .44 & 3.2695947 (0) & 3. $8463422(0)$ & 5. 0081739 (0) & 7.0749911 (0) & 1. 0604277 (1) \\
\hline .45 & 3. $2762632(0)$ & $3.8868412(0)$ & 5. $1289871(0)$ & 7. $3658616(0)$ & 1. 1239660 (1) \\
\hline .46 & $3.2831656(0)$ & $3.9291828 \quad(0)$ & 5. 2568770 & 7. $6782009(0)$ & 1. $1932671 \quad(1)$ \\
\hline .47 & 3. $2903097(0)$ & 3. $9734632(0)$ & 5. 3923515 & $8.0139705(0)$ & 1. 2689713 (1) \\
\hline .48 & 3. $2977036(0)$ & 4. $0197860(0)$ & $5.5359670(0)$ & $8.3753580(0)$ & 1. 3518054 (1) \\
\hline .49 & 3. $3053561(0)$ & 4. $0682633(0)$ & 5. $6883349(0)$ & 8. $7648084(0)$ & 1. 4425963 (1) \\
\hline .50 & 3. $3132763(0)$ & 4. $1190161(0)$ & 5. $8501280(0)$ & 9. 1850601 (0) & 1. 5422873 (1) \\
\hline .51 & $3.3214743(0)$ & 4. 1721755 & $6.0220875 \quad(0)$ & $9.6391874 \quad(0)$ & 1. 6519570 (1) \\
\hline .52 & 3.3299604 (0) & 4. $2278838(0)$ & 6. $2050323(0)$ & 1.0130649 (1) & 1. 7728428 (1) \\
\hline .53 & 3. $3387459(0)$ & 4. $2862954(0)$ & 6. $3998682(0)$ & 1.0663346 (1) & 1. 9063672 (1) \\
\hline .54 & 3. $3478428(0)$ & 4. $3475784(0)$ & 6. 6075997 & 1.1241689 (1) & 2. 0541717 (1) \\
\hline .55 & 3. $3572639(0)$ & 4. $4119159(0)$ & 6. $8293426(0)$ & 1. 1870679 (1) & 2. 2181554 (1) \\
\hline .56 & 3. $3670230(0)$ & 4. $4795076(0)$ & 7. $0663401 \quad(0)$ & 1. 2556002 (1) & 2. 4005228 (1) \\
\hline .57 & 3. $3771348(0)$ & 4. $5505724(0)$ & 7.3199796 (0) & 1. 3304138 (1) & 2. 6038421 (1) \\
\hline .58 & 3. $3876151(0)$ & 4. $6253495(0)$ & 7.5918137 (0) & 1. $4122502(1)$ & 2. 8311150 (1) \\
\hline .59 & 3. $3984809(0)$ & 4. 7041020 & 7.8835849 (0) & 1. 5019596 (1) & 3. 0858639 (1) \\
\hline .60 & 3. $4097506(0)$ & 4. $7871193(0)$ & 8. $1972534(0)$ & 1. 6005209 (1) & 3. 3722376 (1) \\
\hline .61 & $3.4214439(0)$ & 4. $8747204(0)$ & 8. $5350309(0)$ & 1. 7090647 (1) & 3. 6951427 (1) \\
\hline .62 & 3. $4335822(0)$ & 4. $9672582(0)$ & $8.8994209(0)$ & 1.8289016 (1) & 4. 0604078 (1) \\
\hline .63 & 3. $4461887(0)$ & 5. $0651235(0)$ & $9.2932656(0)$ & 1. 9615571 (1) & 4. 4749869 (1) \\
\hline .64 & 3. $4592883(0)$ & 5. $1687509(0)$ & 9. 7198032 (0) & 2.1088134 (1) & 4. 9472173 (1) \\
\hline .65 & 3. $4729085(0)$ & 5. $2786244(0)$ & 1. $0182736(1)$ & 2. 2727623 (1) & 5. 4871440 (1) \\
\hline .66 & 3. $4870789(0)$ & 5. $3952852(0)$ & 1. 0686311 (1) & 2. 4558693 (1) & 6. 1069344 (1) \\
\hline .67 & 3. $5018320(0)$ & 5. $5193397(0)$ & 1. 1235424 (1) & 2. 6610551 (1) & 6. 8214090 (1) \\
\hline .68 & $3.5172034(0)$ & 5. $6514705(0)$ & 1.1835738 (1) & 2.8917967 (1) & 7. 6487274 (1) \\
\hline .69 & 3. $5332319(0)$ & 5. $7924479(0)$ & 1. 2493834 (1) & 3. 1522555 (1) & $8.6112803(1)$ \\
\hline .70 & $3.5499606(0)$ & 5. $9431449(0)$ & 1. 3217398 & 3.4474411 (1) & 9.7368612 (1) \\
\hline
\end{tabular}


TABLE 1.* Values of $\Omega_{\mathrm{j}}(\mathrm{k})$ for $0 \leq \mathrm{j}(1) \leq 9$ and $0 \leq \mathrm{k}^{2}(0.01)<1.00$-Continued

\begin{tabular}{|c|c|c|c|c|c|}
\hline$k^{2}$ & $\Omega_{0}$ & $\Omega_{1}$ & $\Omega_{2}$ & $\Omega_{3}$ & $\Omega_{\mathrm{A}}$ \\
\hline $\begin{array}{l}.71 \\
.72 \\
.73 \\
.74 \\
.75\end{array}$ & $\begin{array}{l}3.5674369(0) \\
3.5857131(0) \\
3.6048476(0) \\
3.6249055(0) \\
3.6459595(0)\end{array}$ & $\begin{array}{l}6.1045547(0) \\
6.2778123(0) \\
6.4642206(0) \\
6.6652823(0) \\
6.8827406(0)\end{array}$ & $\begin{array}{l}1.4015448 \text { (1) } \\
1.4898627(1) \\
1.5879565(1) \\
1.6973345(1) \\
1.8198097(1)\end{array}$ & $\begin{array}{l}3.7834208(1) \\
4.1675905(1) \\
4.6090284(1) \\
5.1189617(1) \\
5.7113855(1)\end{array}$ & $\begin{array}{l}\text { 1. } 1060216(2) \\
1.2625110(2) \\
\text { 1. } 4487127(2) \\
\text { 1. } 6717465(2) \\
1.9408189(2)\end{array}$ \\
\hline $\begin{array}{l}.76 \\
.77 \\
.78 \\
.79 \\
.80\end{array}$ & $\begin{array}{l}3.6680915(0) \\
3.6913941(0) \\
3.7159722(0) \\
3.7419457(0) \\
3.7694524(0)\end{array}$ & $\begin{array}{l}7.1186284(0) \\
7.3753315(0) \\
7.6556690(0) \\
7.9629956(0) \\
8.3013360(0)\end{array}$ & $\begin{array}{ll}1.9575775 & (1) \\
2.1133163 & (1) \\
2.2903221 & (1) \\
2.4926875 & (1) \\
2.7255455 & (1)\end{array}$ & $\begin{array}{l}6.4038975(1) \\
7.2188313(1) \\
8.1848192(1) \\
9.3389738(1) \\
1.0729980(2)\end{array}$ & $\begin{array}{ll}\text { 2. } 2679546 & (2) \\
2.6690316 & (2) \\
\text { 3. } 1652641 & (2) \\
\text { 3. } 7853627 & (2) \\
4.5687313 & (2)\end{array}$ \\
\hline $\begin{array}{l}.81 \\
.82 \\
.83 \\
.84 \\
.85\end{array}$ & $\begin{array}{l}3.7986513(0) \\
3.8297278(0) \\
3.8628993(0) \\
3.8984232(0) \\
3.9366074(0)\end{array}$ & $\begin{array}{l}8.6755606(0) \\
9.0916202(0) \\
9.5568633(0) \\
1.0080473(1) \\
1.0674075(1)\end{array}$ & $\begin{array}{l}2.9954048(1) \\
3.3106179(1) \\
3.6820480(1) \\
4.1240339(1) \\
4.6558190(1)\end{array}$ & $\begin{array}{l}1.2422547 \text { (2) } \\
1.4503942(2) \\
1.7093748(2) \\
2.0358783(2) \\
2.4536454(2)\end{array}$ & $\begin{array}{ll}5.5702880 & (2) \\
6.8678797 & (2) \\
8.5739421 & (2) \\
1.0854291 & (3) \\
1.3959246 & (3)\end{array}$ \\
\hline $\begin{array}{l}.86 \\
.87 \\
.88 \\
.89 \\
.90\end{array}$ & $\begin{array}{l}\text { 3. } 9778240(0) \\
\text { 4. } 0225290(0) \\
\text { 4. } 0712886(0) \\
\text { 4. } 1248185(0) \\
\text { 4. } 1840408(0)\end{array}$ & $\begin{array}{l}1.1352615(1) \\
1.2135628 \text { (1) } \\
1.3049152(1) \\
1.4128679(1) \\
1.5423880(1)\end{array}$ & $\begin{array}{l}5.3037170(1) \\
6.1044816(1) \\
7.1107150(1) \\
8.3998552(1) \\
1.0089734(2)\end{array}$ & $\begin{array}{l}2.9972313(2) \\
3.7182365(2) \\
4.6960084(2) \\
6.0567809(2) \\
8.0095478(2)\end{array}$ & $\begin{array}{ll}1.8276781 & (3) \\
2.4426513 & (3) \\
3.3432588 & (3) \\
4.7056582 & (3) \\
6.8473464 & (3)\end{array}$ \\
\hline $\begin{array}{l}.91 \\
.92 \\
.93 \\
.94 \\
.95\end{array}$ & $\begin{array}{l}\text { 4. } 2501717(0) \\
4.3248613(0) \\
4.4104268(0) \\
4.5102677(0) \\
4.6296635(0)\end{array}$ & $\begin{array}{l}1.7006477(1) \\
1.8984009(1) \\
2.1525401(1) \\
2.4912083(1) \\
2.9650431(1)\end{array}$ & $\begin{array}{l}1.2366829(2) \\
1.5540620 \\
2.0155731 \\
2.7244578 \\
3.8964807\end{array}$ & $\begin{array}{l}1.0917125(3) \\
1.5446583(3) \\
2.2914615(3) \\
3.6165464(3) \\
6.2117605(3)\end{array}$ & $\begin{array}{l}1.0373314(4) \\
1.6516804(4) \\
\text { 2. } 8010734(4) \\
\text { 5. } 1590976(4) \\
1.0636320(5)\end{array}$ \\
\hline $\begin{array}{r}.96 \\
.97 \\
.98 \\
.99 \\
1.00\end{array}$ & $\begin{array}{l}\text { 4. } 7774745(0) \\
\text { 4. } 9703403(0) \\
\text { 5. } 2456872(0) \\
\text { 5. } 7231768(0) \\
\infty\end{array}$ & $\begin{array}{c}3.6752779(1) \\
4.8580263(1) \\
7.2213223(1) \\
1.4303674(2) \\
\infty\end{array}$ & $\begin{array}{cc}6.0473488 & (2) \\
1.0679679 & (3) \\
2.3872660 & (3) \\
9.4878355 & (3) \\
\infty & \end{array}$ & $\begin{array}{c}1.2060257 \text { (4) } \\
2.8419636 \text { (4) } \\
9.5361054(4) \\
\begin{array}{c}\infty .5852838 \\
\infty\end{array}\end{array}$ & $\begin{array}{c}2.5819863(5) \\
8.1144742(5) \\
4.0851238 \\
6.5002882(7) \\
\infty\end{array}$ \\
\hline$k^{2}$ & $\Omega_{5}$ & $\Omega_{6}$ & $\Omega_{7}$ & $\Omega_{8}$ & $\Omega_{9}$ \\
\hline 0.00 & $\pi$ & $\pi$ & $\pi$ & $\pi$ & $\pi$ \\
\hline $\begin{array}{l}.01 \\
.02 \\
.03 \\
.04 \\
.05\end{array}$ & $\begin{array}{l}\text { 3. } 1444016(0) \\
3.1528418(0) \\
3.1669537(0) \\
3.1868051(0) \\
3.2124917(0)\end{array}$ & $\begin{array}{l}3.1454234(0) \\
3,1569389(0) \\
3.1762090(0) \\
3.2033511(0) \\
3.2385310(0)\end{array}$ & $\begin{array}{l}3.1466027(0) \\
3.1616703(0) \\
3.1869086(0) \\
3.2225075(0) \\
3.2687360(0)\end{array}$ & $\begin{array}{l}3.1479395(0) \\
3.1670377(0) \\
3.1990608(0) \\
3.2443006(0) \\
3.3031717(0)\end{array}$ & $\begin{array}{l}\text { 3. } 1494340(0) \\
3.1730429(0) \\
3.2126747(0) \\
3.2687599(0) \\
3.3419109(0)\end{array}$ \\
\hline $\begin{array}{l}.06 \\
.07 \\
.08 \\
.09 \\
.10\end{array}$ & $\begin{array}{l}3.2441379(0) \\
3.2818977(0) \\
3.3259565(0) \\
3.3765321(0) \\
3.4338769(0)\end{array}$ & $\begin{array}{l}\text { 3. } 2819649(0) \\
3.3339215(0) \\
3.3947246(0) \\
3.4647569(0) \\
3.5444634(0)\end{array}$ & $\begin{array}{l}3.3259458(0) \\
3.3945750(0) \\
3.4751540(0) \\
3.5683119(0) \\
3.6747845(0)\end{array}$ & $\begin{array}{l}3.3762170(0) \\
3.4641155(0) \\
3.5676927(0) \\
3.6879319(0) \\
3.8259893(0)\end{array}$ & $\begin{array}{l}\text { 3. } 4329324(0) \\
3.5428347(0) \\
3.6728507(0) \\
3.8244570(0) \\
3.9993993(0)\end{array}$ \\
\hline $\begin{array}{l}.11 \\
12 \\
.13 \\
14 \\
.15\end{array}$ & $\begin{array}{l}3.4982801(0) \\
3.5700701(0) \\
3.6496177(0) \\
3.7373392(0) \\
3.8337006(0)\end{array}$ & $\begin{array}{l}3.6343569(0) \\
3.7350228(0) \\
3.8471259(0) \\
3.9714180(0) \\
4.1087457(0)\end{array}$ & $\begin{array}{l}\text { 3. } 7954235(0) \\
\text { 3. } 9312075(0) \\
\text { 4. } 0832553(0) \\
\text { 4. } 2528403(0) \\
\text { 4. } 4414078(0)\end{array}$ & $\begin{array}{l}3.9832113(0) \\
4.1611549(0) \\
4.3616121(0) \\
4.5866377(0) \\
4.8385832(0)\end{array}$ & $\begin{array}{l}\text { 4. } 1997232(0) \\
\text { 4. } 4278102(0) \\
\text { 4. } 6864215(0) \\
\text { 4. } 9787483(0) \\
\text { 5. } 3084726(0)\end{array}$ \\
\hline $\begin{array}{l}.16 \\
17 \\
.18 \\
19 \\
20\end{array}$ & $\begin{array}{l}\text { 3. } 9392217(0) \\
\text { 4. } 0544809(0) \\
\text { 4. } 1801214(0) \\
\text { 4. } 3168573(0) \\
\text { 4. } 4654806(0)\end{array}$ & $\begin{array}{l}4.2600610(0) \\
4.4264317(0) \\
4.6090547(0) \\
4.8092705(0) \\
5.0285795(0)\end{array}$ & $\begin{array}{l}\text { 4. } 6505957(0) \\
\text { 4. } 8822565(0) \\
\text { 5. } 1384852(0) \\
\text { 5. } 4216493(0) \\
\text { 5. } 7344248(0)\end{array}$ & $\begin{array}{l}5.1201345(0) \\
5.4343576(0) \\
5.7847509(0) \\
6.1753062(0) \\
6.6105807(0)\end{array}$ & $\begin{array}{l}\text { 5. } 6798373(0) \\
\text { 6. } 0977301(0) \\
\text { 6. } 5677814(0) \\
\text { 7. } 0964805(0) \\
\text { 7. } 6913118(0)\end{array}$ \\
\hline $\begin{array}{l}.21 \\
.22 \\
.23 \\
.24 \\
.25\end{array}$ & $\begin{array}{l}\text { 4. } 6268698(0) \\
\text { 4. } 8019991(0) \\
\text { 4. } 9919487(0) \\
\text { 5. } 1979173(0) \\
\text { 5. } 4212351(0)\end{array}$ & $\begin{array}{l}5.2686619(0) \\
5.5313990(0) \\
5.8188984(0) \\
6.1335234(0) \\
6.4779255(0)\end{array}$ & $\begin{array}{l}6.0798376(0) \\
6.4613109(0) \\
6.8827211(0) \\
7.3484615(0) \\
7.8635180(0)\end{array}$ & $\begin{array}{l}7.0957805(0) \\
7.6368586(0) \\
8.2406300(0) \\
8.9149065(0) \\
9.6686559(0)\end{array}$ & $\begin{array}{l}\text { 8. } 3609171(0) \\
9.1152864(0) \\
9.9659856(0) \\
1.0926426(1) \\
1.2012186(1)\end{array}$ \\
\hline $\begin{array}{l}.26 \\
.27 \\
.28 \\
.29 \\
.30\end{array}$ & $\begin{array}{l}5.6633798(0) \\
5.9259941(0) \\
6.2109057(0) \\
6.5201504(0) \\
6.8559986(0)\end{array}$ & $\begin{array}{l}6.8550829(0) \\
7.2683447(0) \\
7.7214814(0) \\
8.2187441(0) \\
8.7649324(0)\end{array}$ & $\begin{array}{l}8.4335554(0) \\
9.0650197(0) \\
9.7652570(0) \\
1.0542653(1) \\
1.1406796(1)\end{array}$ & $\begin{array}{l}1.0512189(1) \\
1.1457381(1) \\
1.2517935(1) \\
1.3709692(1) \\
1.5051001(1)\end{array}$ & $\begin{array}{l}1.3241396(1) \\
1.4635194(1) \\
1.6218282(1) \\
1.8019586(1) \\
2.0073050(1)\end{array}$ \\
\hline $\begin{array}{l}.31 \\
.32 \\
.33 \\
.34 \\
.35\end{array}$ & $\begin{array}{l}\text { 7. } 2209852(0) \\
7.6179448(0) \\
\text { 8. } 0500508(0) \\
\text { 8.5208624 (0) } \\
9.0343771(0)\end{array}$ & $\begin{array}{l}9.3654742(0) \\
1.0026517(1) \\
1.0755037(1) \\
1.1558963(1) \\
1.2447325(1)\end{array}$ & $\begin{array}{l}1.2368671(1) \\
1.3440887(1) \\
1.4637943(1) \\
1.5976553(1) \\
1.7476017(1)\end{array}$ & $\begin{array}{l}1.6563161(1) \\
1.8270952(1) \\
2.0203264(1) \\
2.2393861(1) \\
2.4882297(1)\end{array}$ & $\begin{array}{l}2.2418611 \text { (1) } \\
2.5103359 \text { (1) } \\
2.8182967 \text { (1) } \\
3.1723419 \text { (1) } \\
3.5803135 \text { (1) }\end{array}$ \\
\hline $\begin{array}{l}.36 \\
.37 \\
.38 \\
.39 \\
.40\end{array}$ & $\begin{array}{l}9.5950927 \text { (0) } \\
1.0208078 \text { (1) } \\
1.0879058(1) \\
1.1614509(1) \\
1.2421771(1)\end{array}$ & $\begin{array}{l}1.3430429(1) \\
1.4520056(1) \\
1.5729710(1) \\
1.7074894(1) \\
1.8573456(1)\end{array}$ & $\begin{array}{l}1.9158678(1) \\
2.1050462(1) \\
2.3181518(1) \\
2.5587002(1) \\
2.8308011(1)\end{array}$ & $\begin{array}{l}2.7715029(1) \\
3.0946757(1) \\
3.4642066(1) \\
3.8877418(1) \\
4.3743600(1)\end{array}$ & $\begin{array}{l}\text { 4. } 0515567(1) \\
\text { 4. } 5972404(1) \\
\text { 5. } 2307520(1) \\
5.9681868 \text { (1) } \\
6.8289570(1)\end{array}$ \\
\hline
\end{tabular}

See footnote at end of table, p. 12. 
TABLE 1.* Values of $\Omega_{\mathrm{j}}(\mathrm{k})$ for $0 \leq \mathrm{j}(1) \leq 9$ and $0 \leq \mathrm{k}^{2}(0.01)<1.00$-Continued

\begin{tabular}{|c|c|c|c|c|c|}
\hline$k^{2}$ & $\Omega_{5}$ & $\Omega_{6}$ & $\Omega_{7}$ & $\Omega_{8}$ & $\Omega_{9}$ \\
\hline $\begin{array}{l}.41 \\
.42 \\
.43 \\
.44 \\
.45\end{array}$ & $\begin{array}{l}1.3309183(1) \\
1.4286240(1) \\
1.5363771(1) \\
1.6554164(1) \\
1.7871617(1)\end{array}$ & $\begin{array}{l}2.0245991 \text { (1) } \\
2.2116313 \text { (1) } \\
2.4212044 \text { (1) } \\
2.6565293 \text { (1) } \\
2.9213497(1)\end{array}$ & $\begin{array}{l}3.1392711(1) \\
3.4897718(1) \\
3.8889761(1) \\
4.3447730(1) \\
4.8665169(1)\end{array}$ & $\begin{array}{l}4.9348723 \text { (1) } \\
\text { 5. } 5821923 \text { (1) } \\
6.3317936 \text { (1) } \\
7.2022786 \text { (1) } \\
8.2160859(1)\end{array}$ & $\begin{array}{l}7.8365504(1) \\
9.0194823(1) \\
1.0412490(2) \\
1.2058044(2) \\
1.4008261 \text { (2) }\end{array}$ \\
\hline $\begin{array}{l}.46 \\
.47 \\
.48 \\
.49 \\
.50\end{array}$ & $\begin{array}{l}1.9332450(1) \\
\text { 2. } 0955464(1) \\
2.2762382(1) \\
2.4778370(1) \\
\text { 2. } 7032673(1)\end{array}$ & $\begin{array}{l}3.2200424(1) \\
3.5577398(1) \\
3.9404785(1) \\
4.3753812(1) \\
4.8708801(1)\end{array}$ & $\begin{array}{l}5.4653362(1) \\
6.1545121(1) \\
6.9499504(1) \\
7.8707665(1) \\
8.9400186(1)\end{array}$ & $\begin{array}{l}94003772(1) \\
1.0788151(2) \\
1.2419646(2) \\
1.4344125(2) \\
1.6622140(2)\end{array}$ & $\begin{array}{l}1.6327337 \\
1.9094667 \\
\text { 2. } 2408845 \\
2.6392830 \\
3.1200644(2) \\
3.12)\end{array}$ \\
\hline $\begin{array}{l}.51 \\
.52 \\
.53 \\
.54 \\
.55\end{array}$ & $\begin{array}{l}2.9559372(1) \\
3.2398316(1) \\
3.5596245(1) \\
3.9208178(1) \\
4.3299101(1)\end{array}$ & $\begin{array}{l}5.4369916 \text { (1) } \\
\text { 6. } 0856567 \text { (1) } \\
\text { 6. } 8311633 \text { (1) } \\
7.6906742(1) \\
8.6848881 \text { (1) }\end{array}$ & $\begin{array}{l}1.0185627(2) \\
1.1641533(2) \\
1.3349170(2) \\
1.5359335(2) \\
1.7734580(2)\end{array}$ & $\begin{array}{l}1.9328437(2) \\
2.2555683(2) \\
2.6419288(2) \\
3.1063675(2) \\
3.6670460(2)\end{array}$ & $\begin{array}{l}\text { 3. } 7026116(2) \\
4.4114329 \\
\text { 5. } 2776727 \\
6.3411131 \\
7.6528445 \\
7(2)\end{array}$ \\
\hline $\begin{array}{l}.56 \\
.57 \\
.58 \\
.59 \\
.60\end{array}$ & $\begin{array}{l}\text { 4. } 7946069 \text { (1) } \\
\text { 5. } 3240797(1) \\
5.9292896 \text { (1) } \\
6.6233924(1) \\
7.4222490(1)\end{array}$ & $\begin{array}{l}9.8388739(1) \\
1.1183126(2) \\
1.2754912(2) \\
1.4599991(2) \\
1.6774836(2)\end{array}$ & $\begin{array}{l}2.0552302 \\
2.3908727(2) \\
2.7924109(2) \\
3.2749532(2) \\
3.8575879(2)\end{array}$ & $\begin{array}{l}\text { 4. } 3469219(2) \\
\text { 5. } 1751711(2) \\
6.1890817(2) \\
7.4365906(2) \\
\text { 8. } 9797058(2)\end{array}$ & $\begin{array}{l}9.2788504(2) \\
1.1304851(3) \\
1.3842894(3) \\
1.7040380(3) \\
2.1092534(3)\end{array}$ \\
\hline $\begin{array}{l}.61 \\
.62 \\
.63 \\
.64 \\
.65\end{array}$ & $\begin{array}{l}8.3450710(1) \\
9.4152431(1) \\
1.0661378(2) \\
1.2118673(2) \\
1.3830682(2)\end{array}$ & $\begin{array}{l}\text { 1. } 9349511(2) \\
\text { 2. } 2411423(2) \\
\text { 2. } 6070254(2) \\
\text { 3. } 0464479(2) \\
\text { 3. } 5770065(2)\end{array}$ & $\begin{array}{l}\text { 4. } 5645739(2) \\
\text { 5. } 4269316(2) \\
6.4845852(2) \\
7.7892704(2) \\
9.4085123(2)\end{array}$ & $\begin{array}{l}1.0899157 \\
1.3300762(3) \\
1.6324220 \\
\text { 2. } \\
2.0155347 \\
2.5043262\end{array}$ & $\begin{array}{l}2.6259746 \\
3.2891909 \\
\text { 4. } 1462859 \\
\text { 5. } 2619563 \\
6.7253039\end{array}$ \\
\hline $\begin{array}{l}.66 \\
.67 \\
.68 \\
.69 \\
.70\end{array}$ & $\begin{array}{l}1.5851615(2) \\
1.8249392(2) \\
2.1109677(2) \\
2.4541303(2) \\
2.8683593(2)\end{array}$ & $\begin{array}{l}\text { 4. } 2212147(2) \\
\text { 5. } 0080856(2) \\
\text { 5. } 9752941(2) \\
\text { 7. } 1721622(2) \\
\text { 8. } 6638166(2)\end{array}$ & $\begin{array}{l}1.1431115(3) \\
1.3974809(3) \\
1.7197003(3) \\
2.1310065(3) \\
2.6603265(3)\end{array}$ & $\begin{array}{ll}3.1324761 & (3) \\
3.9459207 & (3) \\
5.0079025 & (3) \\
6.4063588 & (3) \\
8.2648601 & (3)\end{array}$ & $\begin{array}{l}8.6601663(3) \\
1.1240327(4) \\
1.4712160(4) \\
1.9428762(4) \\
2.5902033(4)\end{array}$ \\
\hline $\begin{array}{l}.71 \\
.72 \\
.73 \\
.74 \\
.75\end{array}$ & $\begin{array}{l}\text { 3. } 3716364(2) \\
\text { 3. } 9873716(2) \\
\text { 4. } 7463268(2) \\
\text { 5. } 6893286(2) \\
\text { 6. } 8711446(2)\end{array}$ & $\begin{array}{l}\text { 1. } 0537046(3) \\
\text { 1. } 2908644(3) \\
\text { 1. } 5937432(3) \\
1.9841824(3) \\
\text { 2. } 4925823(3)\end{array}$ & $\begin{array}{l}\text { 3. } 3474663 \text { (3) } \\
\text { 4. } 2478017 \text { (3) } \\
\text { 5. } 4392698 \text { (3) } \\
\text { 7. } 0329383 \\
9.1892247\end{array}$ & $\begin{array}{l}1.0759010(4) \\
1.4141363(4) \\
1.8779832(4) \\
2.5217787 \text { (4) } \\
3.4269672(4)\end{array}$ & $\begin{array}{l}3.4883257(4) \\
4.7489570 \\
6.5405516 \\
9.1209641 \\
1.2891309 \\
1.25 \\
.45\end{array}$ \\
\hline $\begin{array}{l}.76 \\
.77 \\
.78 \\
.79 \\
.80\end{array}$ & $\begin{array}{l}\text { 8. } 3660947 \text { (2) } \\
\text { 1. } 0276297 \\
\text { 1. } 2743971 \\
\text { 1. } 5970122 \\
\text { 2. } 0243431\end{array}$ & $\begin{array}{l}3.1618092(3) \\
\text { 4. } 0531621(3) \\
5.2556423 \\
6.9006308 \\
9.1856068\end{array}$ & $\begin{array}{l}1.2143196(4) \\
1.6244726(4) \\
2.2023463(4) \\
3.0296111(4) \\
4.2347605(4)\end{array}$ & $\begin{array}{l}4.7175815(4) \\
6.5857893 \\
9.3349272 \\
\text { 1. } 3453630 \\
1.9746667 \\
\text { 1. (5) }\end{array}$ & $\begin{array}{l}1.8486494(5) \\
2.6930537(5) \\
3.9909042 \\
6.0258827(5) \\
9.2871234(5)\end{array}$ \\
\hline $\begin{array}{l}.81 \\
.82 \\
.83 \\
.84 \\
.85\end{array}$ & $\begin{array}{ll}2.5985857 & (3) \\
3.3826250 & (3) \\
4.4722115 & (3) \\
6.0166633 & (3) \\
8.2551518 & (3)\end{array}$ & $\begin{array}{l}1.2413348 \text { (4) } \\
\text { 1. } 7058333 \text { (4) } \\
\text { 2. } 3882318 \text { (4) } \\
\begin{array}{l}\text { 3. } 4141676 \text { (4) } \\
\text { 4. } 9972061\end{array}\end{array}$ & $\begin{array}{l}6.0244685(4) \\
8.7393424(4) \\
1.2956045(5) \\
1.9680626(5) \\
3.0728282(5)\end{array}$ & $\begin{array}{l}2.9572117(5) \\
\text { 4. } 5284036(5) \\
7.1085875(5) \\
1.1473581(6) \\
1.9109387(6)\end{array}$ & $\begin{array}{l}1.4640725(6) \\
2.3665859(6) \\
3.9336838(6) \\
6.7461970(6) \\
1.1985330(7)\end{array}$ \\
\hline $\begin{array}{l}.86 \\
.87 \\
.88 \\
.89 \\
.90\end{array}$ & $\begin{array}{l}\text { 1. } 1582518 \text { (4) } \\
\text { 1. } 6673365(4) \\
\text { 2. } 4726623(4) \\
\text { 3. } 7972735 \text { (4) } \\
6.0789976 \text { (4) }\end{array}$ & $\begin{array}{l}7.5129608(4) \\
\text { 1. } 1648160(5) \\
1.8715471(5) \\
\text { 3. } 1357024(5) \\
5.5223607(5)\end{array}$ & $\begin{array}{l}4.9500854(5) \\
8.2655169(5) \\
1.4388026(6) \\
2.6299573(6) \\
5.0951320(6)\end{array}$ & $\begin{array}{l}3.2984010(6) \\
5.9314923(6) \\
1.1186011(7) \\
2.2306362(7) \\
4.7538466(7)\end{array}$ & $\begin{array}{l}2.2165832(7) \\
4.2928225(7) \\
8.7706067(7) \\
1.9080277(8) \\
4.4730772(8)\end{array}$ \\
\hline $\begin{array}{l}.91 \\
.92 \\
.93 \\
.94 \\
.95\end{array}$ & $\begin{array}{l}1.0234053(5) \\
\text { 1. } 8334510(5) \\
\text { 3. } 5540054(5) \\
\text { 7. } 6378369(5) \\
\text { 1. } 8898335(6)\end{array}$ & $\begin{array}{l}\text { 1. } 0330799(6) \\
\text { 2. } 0822980(6) \\
\text { 4. } 6133600(6) \\
\text { 1. } 1567756(7) \\
\text { 3. } 4349093(7)\end{array}$ & $\begin{array}{l}1.0591208 \text { (7) } \\
2.4017606(7) \\
6.0816052(7) \\
1.7791735(8) \\
6.3399610(8)\end{array}$ & $\begin{array}{l}1.0980174(8) \\
2.8013155(8) \\
8.1069617(8) \\
2.7670704(9) \\
1.1832720(10)\end{array}$ & $\begin{array}{l}1.1479956(9) \\
3.2950159(9) \\
1.0898261(10) \\
4.3398773(10) \\
2.2270714(11)\end{array}$ \\
\hline $\begin{array}{r}.96 \\
.97 \\
.98 \\
.99 \\
1.00\end{array}$ & $\begin{array}{c}\text { 5. } 7351988(6) \\
\text { 2. } 4035009(7) \\
\text { 1. } 8152204(8) \\
\text { 5. } 7774227(9) \\
\infty\end{array}$ & $\begin{array}{l}\text { 1. } 3031098 \text { (8) } \\
\text { 7. } 2819128 \text { (8) } \\
\text { 8. } 2499417 \text { (9) } \\
\text { 5. } 2518697 \text { (11) } \\
\infty\end{array}$ & $\begin{array}{cc}3.0066486 & (9) \\
2.2402975 & (10) \\
3.8073399 & (11) \\
4.8476752 & (13) \\
\infty & \end{array}$ & $\begin{array}{cc}\text { 7. } 0146360 & (10) \\
6.9691688 & (11) \\
\text { 1. } 7766503 & (13) \\
\text { 4. } 5243605 & (15) \\
\infty & \end{array}$ & $\begin{array}{cc}1.6503480 & (12) \\
2.1862541 & (13) \\
8.3603249 & (14) \\
4.2581255 & (17) \\
\infty & \end{array}$ \\
\hline
\end{tabular}

*The figures in parentheses indicate the power of ten by which the adjacent entry is to be multiplied; e.g., $1.0412490(2)=104.12490$.

TABle 2. Simple power series (eq 2.1-4)

These $m$-values are a lower bound on the number of terms to sum for $\Omega_{j}(k)$ to ten significant figures.

\begin{tabular}{l|c|c|c|c}
\hline \hline & \multicolumn{2}{|c|}{$j=0$} & \multicolumn{2}{|c}{$j=8$} \\
\cline { 2 - 4 } & $k^{2}=0.01$ & $k^{2}=0.99$ & $k^{2}=0.01$ & $k^{2}=0.99$ \\
\cline { 2 - 3 } & & & & \\
\hline $\begin{array}{c}\text { Minimum } \\
\alpha_{m}(j) k^{4} m / \Omega_{j}(k)<10^{-11} \ldots \text { value for which }\end{array}$ & 3 & 823 & 4 & 4615 \\
\hline
\end{tabular}


TABLE 3. Difference sum (eq 2.2-6)

The contribution of the difference sum to the total $\Omega_{j}(k)$ is here compared with the contribution from the closed-form apnroximation. The $m$-values at which difference-sum terms contribute less than a part in $10^{11}$ may be compared with $m$-values in table 2 for the simple power series.

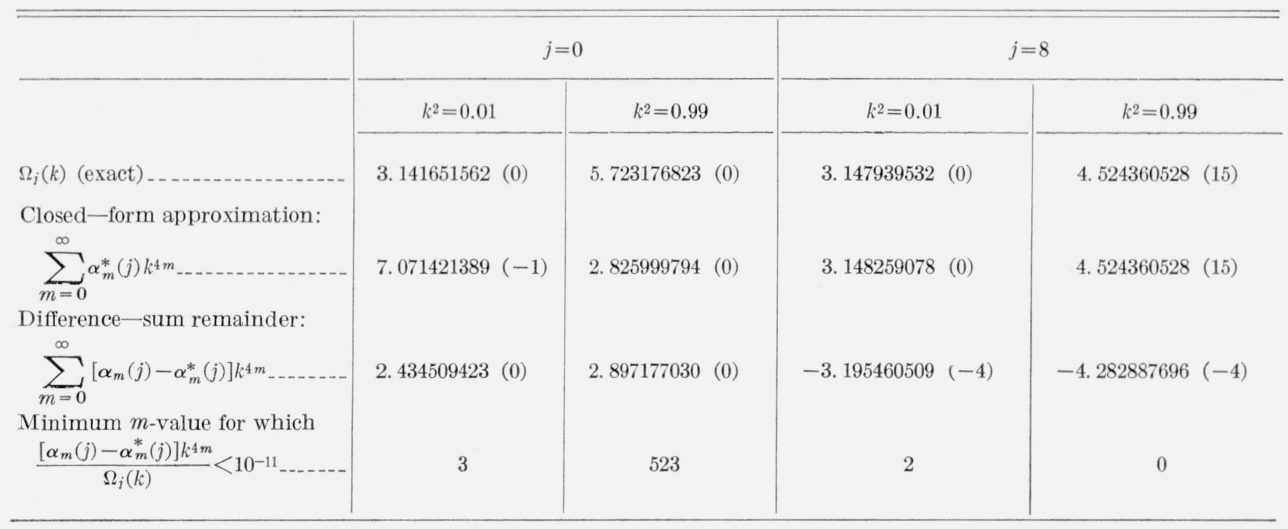

TABLE 4. Bessel function formulation for $\Omega_{\mathrm{j}}(k)$ (eqs 2.3-27, 28)

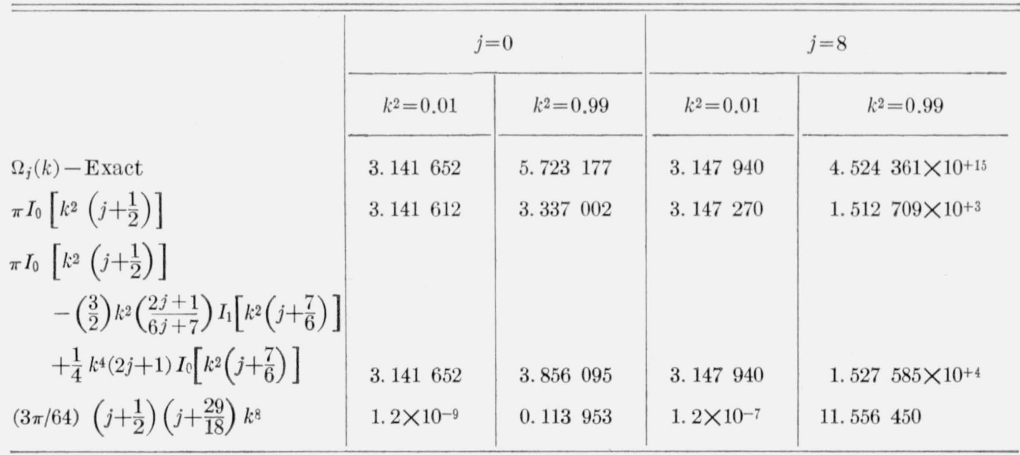

TABLE 5. Error function formulation for $\Omega_{\mathrm{j}}(\mathrm{k})($ eq $2.4-41)$

\begin{tabular}{|c|c|c|c|c|}
\hline & \multicolumn{2}{|c|}{$j=0$} & \multicolumn{2}{|c|}{$j=8$} \\
\hline & $k^{2}=0.01$ & $k^{2}=0.99$ & $k^{2}=0.01$ & $k^{2}=0.99$ \\
\hline$\Omega_{j} /(k)$-Exact & 3. 141652 & 5. 723177 & 3. 147940 & 4. $524361 \times 10^{+15}$ \\
\hline $\begin{array}{l}\sqrt{\pi /(2 j+1) k^{2}}\left(1-k^{2}\right)^{-j} \text { erf } \lambda \\
\text { 1st } 2 \text { terms in } 2.4-41 \\
\text { All } 3 \text { terms in } 2.4-41\end{array}$ & $\begin{array}{l}\text { 3. } 131384 \\
\text { 3. } 144486 \\
\text { 3. } 140552\end{array}$ & $\begin{array}{r}1.781883 \\
3.121919 \\
-1.354046\end{array}$ & $\begin{array}{ll}\text { 2. } 994 & 801 \\
\text { 3. } 177 & 138 \\
\text { 3. } 159 & 417\end{array}$ & $\begin{array}{l}\text { 3. } 822991 \times 10^{+15} \\
\text { 3. } 992220 \times 10^{+15} \\
\text { 3. } 958982 \times 10^{+15}\end{array}$ \\
\hline
\end{tabular}

TABLE 6. Numerical integration technique (eqs 2.5, 44-49)

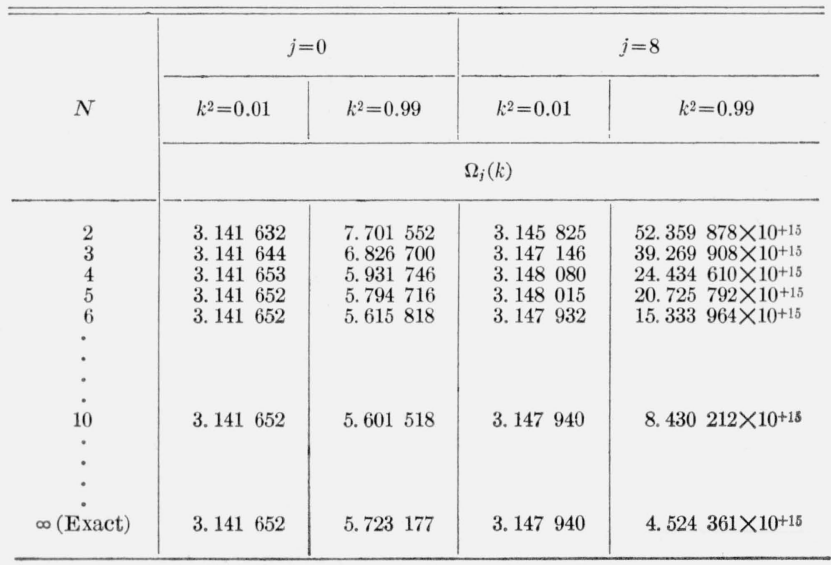


Table 2, which applies to what has been called the simple power series above, illustrates how rapidly the number of terms required increases as either $k$ or $j$ goes up. These figures were obtained by setting the $m$ 'th term in eq. (2.1-4) equal to $\Omega_{j}(k) \times 10^{-11}$ and solving for $m .^{5}$ For large $j$ and $k$ values, the use of this formulation becomes almost prohibitively laborious.

In table 3, where the difference sum technique is examined, a number of interesting characteristics of this formulation are apparent. For small values of $k$, the improvement over the simple power series is negligibly small for all values of $j$. For $k$ approaching unity, there is a considerable reduction in the labor involved to obtain any predetermined accuracy. For $j=0, k^{2}=0.99$ to obtain a maximum of ten significant figures, note that the number of terms required is cut from 823 to 523 . While this improvement is not as spectacular as some cases which have been examined [8], it is by no means unimportant. The real gain in using this technique is seen at $j=8, k^{2}=0.99$; here we see that the closed-form approximation alone gives $\Omega_{j}(k)$ correct to one part in $10^{19}$. This can be compared with the more than 4615 terms indicated in table 2 for ten or less significant figures using the unmodified power series.

To check the behavior of the Bessel function formulation, section 2.3 above, the BAAS tables [16] of $I_{0}(x)$ and $I_{1}(x)$ were used along with suitable interpolation tables [14]. The results are given in table 4 . It will be recalled that these relations were derived essentially by approximating only the first few terms of the integrand in eq $2-1$. For this reason, the formulation as would be expected yields excellent values of $\Omega_{j}(k)$ when $k^{2}=0.01$ at both $j=0$ and $j=8$; but for $k^{2}$ near unity, this technique of calculation is entirely inadequate.

The error function formulation [17], section 2.4, table 5, is also based on approximations to only the first few terms in the expansion of the integrand. Note that the leading term from 2.4-41 is not a bad approximation to the value of $\Omega_{j}(k)$; but the inclusion of the second or third terms results in little or no improvement. Evidently many more terms are needed to obtain useful results by this technique.

The importance of the erf and $I_{n}$ formulations of $\Omega_{j}(k)$ arises, then, not so much for their usefulness in numerical computation, as from the relations they demonstrate between the new transcendental function considered here and older, more familiar forms.

The numerical integration formulas, from section 2.4, are quite different in kind. These, it will be observed from table 6 , give excellent agreement for small $k$ values, although they leave something to be desired for $k$ near unity. It is interesting to note that, at these selected points, the accuracy of the formulas does not increase uniformly as $N$ increases-for example, at $k^{2}=0.99, j=0$, the formulas with $N=5$ and 6 are virtually equivalent, and each is better than $N=10$. The approximation for large $j k^{2}$ is excellent. Note that by substituting the value of $\Omega_{j}(k)$ at $k^{2}=0.99$ and $j=8$ from table 1 it may be concluded that

$$
\lim _{N \rightarrow \infty} w_{N}(0) / \sum_{i=0}^{N} w_{N}(i)=\lim _{N \rightarrow \infty} \frac{(-1)^{N}}{N \cdot N !} \int_{0}^{N}(t-1)(t-2)(t-3) \ldots(t-N) d t \cong 0.0144 \ldots
$$

It thus appears that there is a multiplicity of choices available for computing $\Omega_{j}(k)$ for small $k$ values, and for $k$ approaching unity the recursion formula (1-7) can be used with the appropriate high- $k$ expansions $(1-10,11)$ for ordinary elliptic integrals. However, for highprecision coverage of the entire range $0 \leq k<1$ with a single formulation, only the differencesum technique appears to be practical.

${ }^{5}$ It should be noted that, since (2.1-4) is not an alternating series, the solution for $m$ of the inequality

$$
\alpha_{m}(j) k^{4 m} \leq \Omega_{j}(k) \times 10^{-11}
$$

serves only as a general indication of the convergence rate and does not necessarily imply that the remainder

$$
R_{m}(j, k)=\sum_{m+1}^{\infty} \alpha_{m}(j) k^{4 m}
$$

is less than $\Omega_{j}(k) \times 10^{-11}$. It does, however, indicate the minimum number of terms to sum, beyond which there exists, for $k^{2}<1$, a value $m^{\prime} \geq m$ for which

$$
R_{m \prime} \geq^{m}(j, k) \leq \Omega_{j}(k) \times 10^{-11}
$$


The authors thank R. J. Herbold for programing and computing table 1, R. L. Bach for numerical checks by alternative formulations, L. C. Maximon for suggesting Stirling's formula as a basis for the difference-sum, and M. J. Berger for other helpful suggestions in the completion of this work.

\section{Appendix A. Evaluation of $\alpha_{m}^{*}(j)$}

Stirling's formula $[11$, p. 5] may be written in the form

$$
z !=\sqrt{2 \pi z} z^{z} e^{-z} H(z)
$$

in which $H(z)$ can be represented by the asymptotic expansion [18]

$$
\lim _{z \rightarrow \infty} H(z)=1+1 /(12 z)+1 /\left(288 z^{2}\right)-139 /\left(51840 z^{3}\right)-\ldots
$$

In order to use formula (A1) above, it is convenient to write eq $(2-5)$ as

$$
\alpha_{m}(j)=\frac{\pi}{(64)^{m}} \frac{(4 m) !}{(2 m) !(m !)^{2}} \prod_{\nu=1}^{j}\left(\frac{4 m}{2 \nu-1}+1\right)
$$

in which the product is to be taken as unity for $j=0$. Substitution of (A1) for the factorials in (A3) gives

$$
\alpha_{m}(j)=(1 / m \sqrt{2}) \frac{H(4 m)}{H(2 m) H^{2}(m)} \prod_{\nu=1}^{j}\left(\frac{4 m}{2 \nu-1}+1\right) .
$$

By using (A2) for the $H$ terms, and expressing the finite product as a $j$ th degree polynomial in $m$, it follows that

$$
\begin{aligned}
\lim _{m \rightarrow \infty} \alpha_{m}(j)=(1 / m \sqrt{ } 2) & {\left[1-\frac{3}{16}\left(\frac{1}{m}\right)+\frac{9}{512}\left(\frac{1}{m}\right)^{2}+39\left(\frac{1}{m}\right)^{3}+\ldots\right] \times\left[1+m \cdot 4 \sum_{\nu=1}^{j} \frac{1}{2 \nu-1}\right.} \\
& +m^{2} \cdot 8 \sum_{\nu=1}^{j}\left\{-\frac{1}{(2 \nu-1)^{2}}+\frac{1}{(2 \nu-1)} \sum_{\mu=1}^{j} \frac{1}{2 \mu-1}\right\}+\ldots \\
& \left.+m^{j-2} \cdot 2^{+3 j} \frac{j !}{(2 j) !} \cdot \frac{1}{96} j(j-1)\left(3 j^{2}-j-1\right)+m^{j-1} \cdot 2^{+3 j} \frac{j !}{(2 j) !} \cdot \frac{1}{4} j^{2}+m^{j} \cdot 2^{+3 j} \frac{j !}{(2 j) !}\right] \\
=(1 / \sqrt{2}) \sum_{i=0} & C_{i}(j) m^{j-i-1}
\end{aligned}
$$

where

$$
\begin{aligned}
& C_{0}(j)=+2^{+3 j} \frac{j !}{(2 j) !} \\
& C_{1}(j)=+2^{+3 j} \frac{j !}{(2 j) !} \cdot \frac{1}{16}\left(4 j^{2}-3\right) \\
& C_{2}(j)=+2^{+3 j} \frac{j !}{(2 j) !} \cdot \frac{1}{1536}\left(48 j^{4}-64 j^{3}-72 j^{2}+16 j+27\right)
\end{aligned}
$$

and also

$$
C_{j}(j)=2^{-3 j}\left(\begin{array}{c}
2 j \\
j
\end{array}\right)
$$


which can be established by methods of mathematical induction. Now it is possible to show that $m^{n}$ can be expressed in terms of binomial coefficients, in the form

$$
m^{n}=\sum_{k=0}^{n}\left(\begin{array}{c}
m+k \\
m
\end{array}\right) \beta_{n, k}
$$

where the $\beta_{n, k}$ 's are related to Kummer's numbers [19]. It can readily be shown that

$$
\beta_{n, 0}=(-1)^{n} \text { and } \beta_{n, n}=n !
$$

\begin{tabular}{|c|c|c|c|c|c|}
\hline$k$ & 0 & 1 & 2 & 3 & 4 \\
\hline $\begin{array}{l}0 \\
1 \\
2 \\
3 \\
4\end{array}$ & +1 & $\begin{array}{l}-1 \\
+1 \\
\end{array}$ & $\begin{array}{l}+1 \\
-3 \\
+2\end{array}$ & $\begin{array}{l}-1 \\
+7 \\
-12 \\
+6\end{array}$ & $\begin{array}{l}+1 \\
-15 \\
+50 \\
-60 \\
+24\end{array}$ \\
\hline
\end{tabular}

A few values of these constants are tabulated below

Now ignoring terms of the order of $1 / m^{2}$ in the expression $(\mathbf{A}-5)$, for large $m$ values, $\alpha_{m}(j)$ can be approximated by the expression

$$
(1 / \sqrt{2})\left[\sum_{i=0}^{j-1} C_{i}(j) m^{j-i-1}+C_{j}(j) m^{-1}\right]
$$

Replacing $1 / m$ by its equivalent to this order, $1 /(m+1)$, and using $(\mathrm{A}-7)$ in the sum term, it follows that

$$
\begin{aligned}
\alpha_{m}^{*}(j) & =(1 / \sqrt{2})\left[\sum_{i=0}^{j-1} C_{i}(j) \sum_{k=0}^{j-i-1} \beta_{j-i-1, k}\left(\begin{array}{c}
m+k \\
m
\end{array}\right)+C_{\jmath}(j) \frac{1}{m+1}\right] \\
& =(1 / \sqrt{2}) 2^{-3 j}\left(\begin{array}{c}
2 j \\
j
\end{array}\right)\left\{\frac{1}{m+1}+\sum_{i \geq 0}^{j-1} B_{i j}\left(\begin{array}{c}
m+j-i-1 \\
m
\end{array}\right)\right\}
\end{aligned}
$$

which is eq 2.2-8 of the text above. The $B_{i j}$ expression in eq $(\mathrm{A}-8)$ is obtained by combining the $C_{i}(j)$ and $\beta_{n, k}$ values as indicated and factoring out $C_{j}(j)$. The expression $(2.2-11)$ above is then obtained using the familiar series

and

$$
\ln (1-x)=-\sum_{m=0}^{\infty} x^{m+1} /(m+1)
$$

$$
(1-x)^{-k-1}=\sum_{m=0}^{\infty}\left(\begin{array}{c}
m+k \\
m
\end{array}\right) x^{m}
$$

\section{References}

[1] J. H. Hubbell, R. L. Bach, and R. J. Herbold, Radiation field from a circular disk source, J. Research NBS 65C (Engr. and Instr.) No. 4,249 (1961). In this paper, the notation $\mathrm{S}_{n}(k)$ is used for the function which here is designated as $\Omega_{j}(k)$. 
[2] M. J. Berger and J. C. Lamkin, Sample calculations of gamma-ray penetration into shelters: Contributions of sky shine and roof contamination, J. Research NBS $\mathbf{6 0}, 109$ (1958) RP2827.

[3] P. F. Byrd and M. D. Friedman, Handbook of Elliptic Integrals for Engineers and Physicists (Springer, Berlin, 1954).

[4] B. O. Peirce, A Short Table of Integrals, 4th Ed., revised by R. M. Foster, (Ginn, New York, 1957).

[5] H. B. Dwight, Tables of Integrals and Other Mathematical Data, 3rd Ed. (MacMillan, New York, 1957).

[6] A. Fletcher, Guide to tables of elliptic functions, M.T.A.C. 3, 229 (1948).

[7] C. Hastings, Jr., Approximations for Digital Computers (University Press, Princeton, 1955).

[8] L. F. Epstein and N. E. French, Improving the convergence of series: Application to some elliptic integrals, Am. Math. Monthly 63, 698 (1956).

[9] E. P. Adams, Ed., Smithsonian Mathematical Formulae and Tables of Elliptic Functions (Smithsonian Institution, Washington, 1957), 3d Reprint.

[10] C. Jordan, Calculus of Finite Differences (Chelsea Publ. Co., New York, 1947) 2d ed., p. 69.

[11] E. Jahnke, and F. Emde, Tables of Higher Functions, 6th Edition, Friedrich Losch, Ed. (McGraw-Hill, New York, 1960).

[12] G. N. Watson, Theory of Bessel Functions, 2nd Ed. (University Press, Cambridge, 1944).

[13] K. Pearson, Tables of the Incomplete Gamma Function (University Press, Cambridge, 1957).

[14] Mathematical Tables Project, WPA, Tables of Lagrangian Interpolation Coefficients. 2d Printing. (Columbia University Press, New York, 1948).

[15] E. T. Whittaker and G. Robinson, The Calculus of Observations, 4th Ed. (Blackie, London, 1958).

[16] British Association for the Advancement of Science, Mathematical Tables, Volume VI. Bessel Functions, Part I. (University Press, Cambridge, 1958).

[17] National Bureau of Standards, Tables of the error function and its derivatives, Applied Mathematics Series, No. 41 (Washington, 1954).

[18] For coefficients of this expansion through the $1 / z^{7}$ terms, see H. T. Davis, Tables of the Higher Mathematical Functions, Volume I, p. 180. (Principia Press, Bloomington, Indiana, 1933.)

[19] E. E. Kummer, J. F. Math. (Crelle) 21, 74 (1840), See also Fletcher, A., Miller, J. C. P., Rosenhead, L. and Comrie, L. J.: An Index of Mathematical Tables, 2d Ed. (Addison-Wesley, Reading, Mass., 1962) vol. I, p. 108.

(Paper 67B1-88) 